\title{
Ist hoher Leistungsdruck nachhaltig?
}

\section{Liebe Leserinnen und Leser,}

wie kann das Controlling dazu beitragen, Leistungsreserven im Management zu mobilisieren? Wie kann internes Unternehmertum - auch in großen Unternehmen - gefördert werden? Wie stellt man sicher, dass sich hinter Organigramm und Budgetierung keine zu großen Komfortzonen entwickeln? Die Antwort auf diese Fragen ist nicht einfach, und es gibt sicherlich keinen Königsweg. Mit dem vorliegenden Heft stellen wir Ihnen aber eine Reihe von Bausteinen des Performance Managements vor, die einige Unternehmen zu genau diesem Zweck einsetzen: anspruchsvolle und dominant top-down gesetzte Ziele, ein hohes Maß an Leistungstransparenz, eine Kultur des offenen Informationsaustausches und der konstruktiven Kritik und nicht zuletzt eine relative Bewertung des Management Teams mit klaren Sanktionen für die im Vergleich schlecht abschneidenden Führungskräfte. Solche auf Höchstleistungen und internes Unternehmertum zielenden Konzepte haben viele Väter - vom amerikanischen Management-Guru Jack Welch über die Autoren des im Gesamtpaket bislang wenig erfolgreichen Buchs „Beyond Budgeting“ bis hin zu Robert Simons aus Harvard. In Deutschland ist aktuell Henkel ein prominentes Beispiel für ein Unternehmen, das sich konsequent einer "Winning Culture“ und dem damit verbundenen Streben nach Höchstleistungen verschrieben hat. Wir sind daher besonders stolz darauf, dass wir im vorliegenden Heft dieses Themenfeld im Dialog mit Kasper Rorsted, dem Vorstandsvorsitzenden der Henkel KGaA, erörtern können. Seine sieben Jahre als Vorstandsvorsitzender der Henkel KGaA sind eine Erfolgsgeschichte: Als er 2008 eine bereinigte EBIT-Marge von 14 Prozent für 2012 anvisierte, erschien dies den meisten Analysten (und vermutlich auch den meisten Henkelianern) als wenig realistisch. Allein, Kasper Rorsted hat sein Ziel trotz der Finanzkrise realisiert und seitdem nicht damit aufgehört, dem Unternehmen neue, ehrgeizige Ziele zu setzen und diese dann auch zu erreichen.

Bei allem Respekt vor solchen Erfolgen: Hat eine immer intensivere Mobilisierung der Leistungsreserven großer Teile des Managements nicht auch eine Schattenseite, die es zu

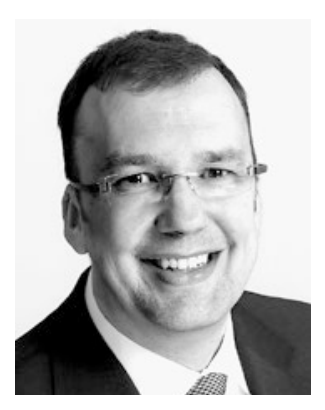

Utz Schäffer

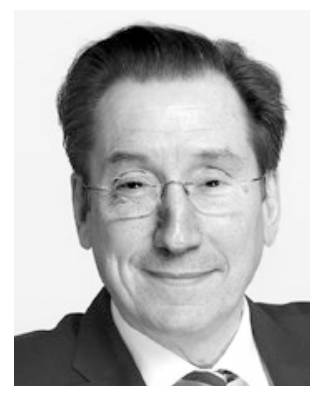

Jürgen Weber

adressieren gilt? Wir denken ja. Zum einen überschreiten Menschen, die unter hohem Druck stehen, mit höherer Wahrscheinlichkeit die Grenzen des Erlaubten oder loten zumindest die Graubereiche intensiver aus. Top Management, Revision und Controlling gemeinsam müssen daher die Spielregeln noch eindringlicher kommunizieren und klare Grenzen setzen. Compliance muss mehr sein als ein Papiertiger. Zum anderen stellt sich die Frage, welche Arbeitsbelastung über welchen Zeitraum nachhaltig und gesund ist - individuell wie kollektiv. Wir beobachten, dass nicht nur in Unternehmensberatungen, Investment-Banken und Gründungsfabriken eine ausgeglichene Balance zwischen Arbeit und Privatleben häufig fehlt, sondern dass auch im Management großer Konzerne die durchschnittlich geleisteten Stunden immer mehr werden. Wie nachhaltig und gesund das ist, wird die Zukunft zeigen. Ein spannendes Experiment.

Viel Vergnügen bei der Lektüre wünschen Ihnen

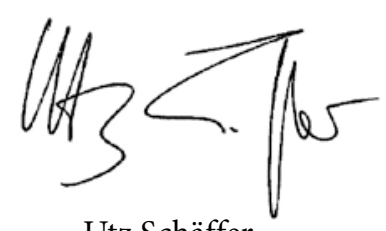

Utz Schäffer

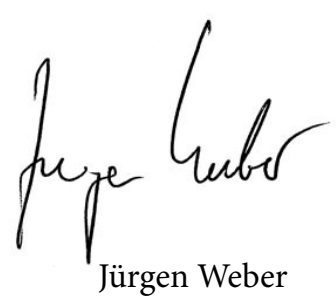

